\title{
Subarachnoid Hemorrhage in Perivascular Spaces Mimicking Brainstem Hematoma
}

\author{
Manuel Ribeiro, Peter Howard, Robert A. Willinsky, Karel ter Brugge, Leodante da Costa
}

Can. J. Neurol. Sci. 2010; 37: 286-288

Aneurysmal subarachnoid hemorrhage (SAH) can be devastating and associated intraparenchymal hematomas significantly increase the likelihood of neurological deficit. We report a case of a patient with an aneurysmal subarachnoid hemorrhage and a brainstem hematoma, but suffering only minimal sensory deficits. Our hypothesis is that the apparent brainstem hematoma was actually subarachnoid blood in dilated Virchow-Robin spaces. To our knowledge, this is the first report of subarachnoid hemorrhage extending into Virchow-Robin spaces.

\section{CASE RePort}

A 60-year-old female was admitted with SAH. Neurological examination revealed a drowsy but easily rousable patient, oriented, without motor deficits. Computerized tomography (CT) revealed blood in the basal cisterns and Sylvian fissures, intraventricular hemorrhage, hydrocephalus and a hematoma in the ponto-mesencephalic junction (Figure 1). After external ventricular drainage, the Glasgow Coma Scale improved to 15. A computed tomography angiogram revealed three aneurysms: a larger, irregular basilar tip (Figure 1-D), a smaller left posterior communicating artery aneurysm and an extradural small right paraclinoid aneurysm. Both intracranial lesions were treated with endovascular coiling. Persistent perioral hypo-esthesia was noted in follow-up neurological examination. Magnetic Resonance Imaging three weeks after coiling showed residual blood in the brainstem with mild edema and enlarged VirchowRobin spaces in the midbrain, basal ganglia, insula and deep white matter over the convexities (Figure 2).

\section{Discussion}

Subarachnoid haemorrhage is the usual presentation of intracranial aneurysms ${ }^{1}$. Intraparenchymal hematomas can occur, being more common with middle cerebral artery ${ }^{2}$ and rare with posterior fossa aneurysms ${ }^{3}$, where intraventricular bleeding without parenchymal haemorrhage is the usual pattern ${ }^{4}$. The presence of intraparenchymal brainstem hematomas is not common and has not been described in a patient with a good clinical grade.

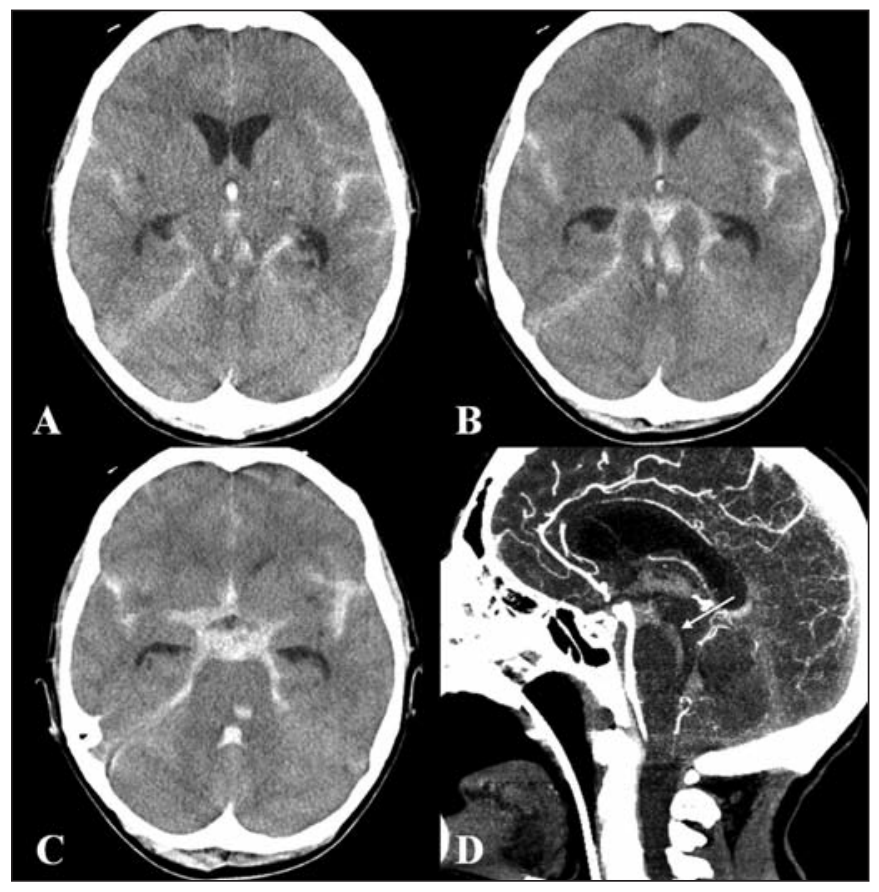

Figure 1: Initial pre-contrast axial CT scan ( $A, B$ and $C$ ) shows subarachnoid hemorrhage, intraventricular hemorrhage, and hydrocephalus. There is a hematoma in the pontomesencephalic region bilaterally. Sagittal reformat of CT angiogram (D) shows the basilar tip aneurysm, and demonstrates the cranio-caudal extent of the apparent brainstem hematoma (arrow).

The presence of intraparenchymal hematoma after aneurysm rupture is associated with poor prognosis ${ }^{2,3}$. The topography of intracerebral haemorrhage is closely related to the location of the aneurysm ${ }^{2,3,5,6}$. Aneurysm rupture and concurrent hypertensive hemorrhage into the brain parenchyma might be considered in the differential diagnosis. This association is extremely rare ref $^{6,7}$ and in our case seems unlikely, taking into account the location and pattern of the bleeding. Duret hemorrhage, another

From the Division of Neuroradiology, Department of Radiology (MR), Hospital São Marcos, Braga, Portugal; Department of Medical Imaging (RAW, KtB), Toronto Western Hospital; Department of Medical Imaging (PH), Division of Neurosurgery, Department of Surgery (LdC), Sunnybrook Health Sciences Centre, University of Toronto, Ontario, Canada.

Received July 24, 2009. Final Revisions Submitted September 2, 2009.

Correspondence to: Leodante da Costa, Sunnybrook Health Sciences Centre, 2075 Bayview Avenue, Room A1-37, Toronto, Ontario, M4N 5M3, Canada. 


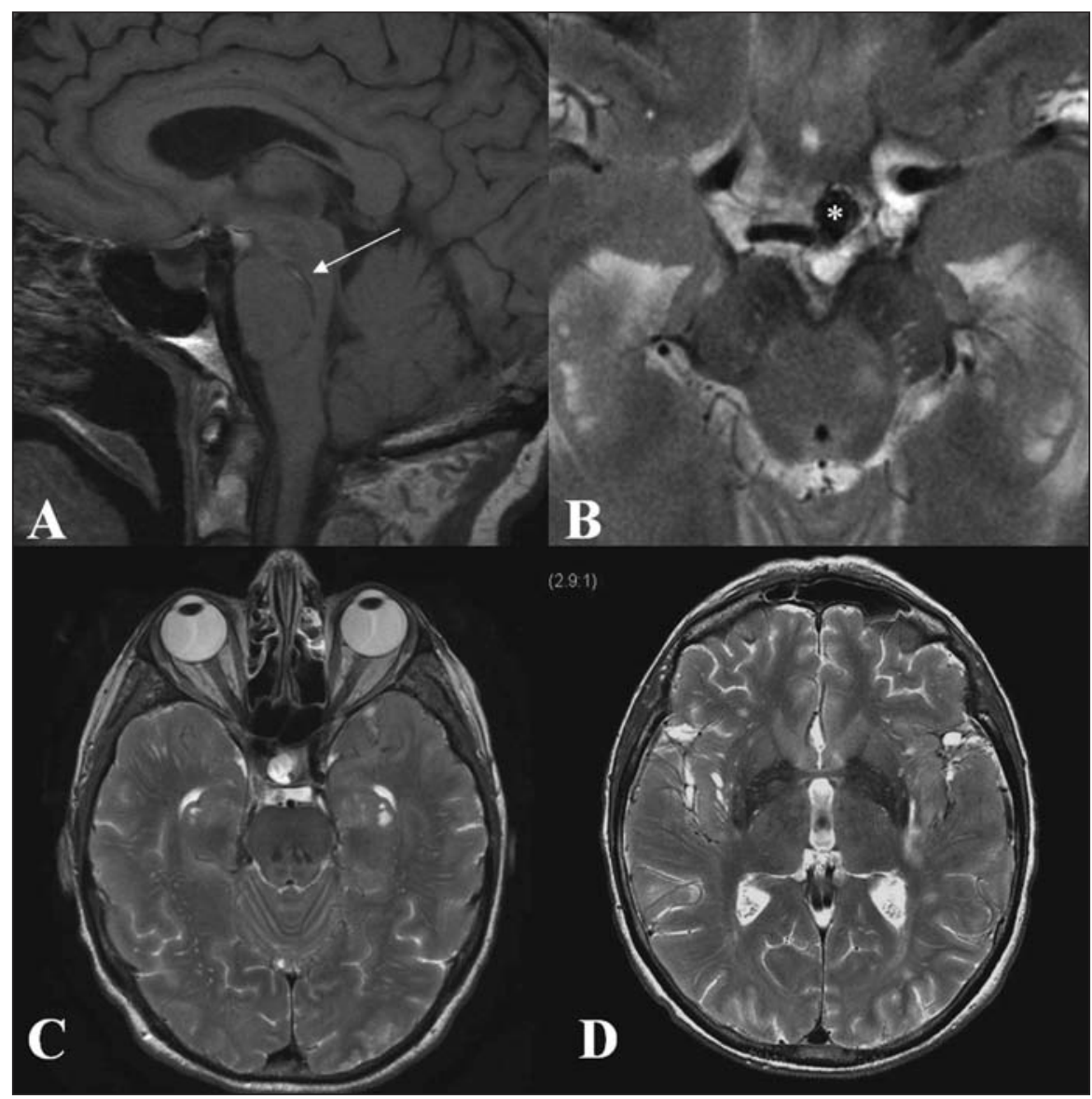

Figure 2: MR study: sagittal T1 (A) shows evolution of the vertically elongated brainstem hematoma with central methemoglobin and hemosiderin rim (long arrow). Axial T2 (B) demonstrates prominence of the perivascular spaces involving the midbrain and loculated subarachnoid blood (B) adjacent to coiled aneurysm (asterisk). Axial T1 (C) and T2 (D) showing bilateral residual pontomesencephalic hematoma.

differential diagnosis was excluded due to the absence of signs of raised intracranial pressure and transtentorial herniation ${ }^{8}$.

Our hypothesis for the apparent brainstem hematoma is that the SAH extended into existing perivascular spaces, eventually dissecting cranio-caudally along fibre tracts within the brainstem. Perivascular enlargements, also known as VirchowRobin spaces, are pial-lined interstitial fluid spaces surrounding penetrating arteries, often found in the basal ganglia and the superior centrum semiovale ${ }^{9,10}$. In the midbrain they can follow the path of branches of the collicular and accessory collicular arteries penetrating the pontomesencephalic junction, closely following the ponto-mesencephalic sulcus ${ }^{11}$. The disposition of the hemorrhage, similar to the usual location of Virchow-Robin spaces, between the cerebral peduncle and the subtantia nigra ${ }^{12}$, is consistent with this hypothesis. Despite the absence of direct communication between the cisterns of the subarachnoid space and the perivascular spaces ${ }^{9,13,14}$, thin pial membranes separating them could be torn by the high pressure jet from the ruptured aneurysm.
Brainstem hemorrhage is usually associated with high mortality and neurological morbidity ${ }^{15,16}$. Considering the radiological picture, a poor clinical grade should be expected. However, after the external ventricular drain was inserted, the patient's only neurological deficit was mild peri-oral paresthesias. "Pure" sensory symptoms caused by brainstem strokes have been reported, mostly related with small dorsally located ischemic infarcts. The word "pure" may be a misnomer, once other minor brainstem symptoms such as dizziness and gait ataxia are often associated ${ }^{17}$. A bilateral trigeminal lesion, involving the ascending trigeminal secondary tract (trigeminal lemniscus) located medially in the medial lemniscus ${ }^{18}$ may be the explanation for the bilateral peri-oral paresthesia in our patient. The paucity of neurological signs from the hemorrhage seen on the initial CT reinforces our hypothesis of pre-existing Virchow-Robin spaces. 


\section{Conclusion}

Aneurysmal subarachnoid hemorrhage can be devastating, and the presence of an intraparenchymal hematoma increases the probability of neurological deficit. We present a case of an apparent brainstem hemorrhage secondary to intracranial aneurysm rupture with a discordantly mild clinical presentation characterized by mild sensory deficit, probable explained by the hemorrhage extending into enlarged Virchow-Robin spaces in the brainstem with minimal impact on the surrounding parenchyma. This is the first case of such occurrence and emphasizes the importance of aggressive resuscitation and a proper clinical examination in face of an alarming imaging finding during management of SAH.

\section{REFERENCES}

1. Kassell NF, Torner JC, Haley EC, Jr., Jane JA, Adams HP, Kongable GL. The International Cooperative Study on the Timing of Aneurysm Surgery. Part 1: Overall management results. J Neurosurg. 1990;73(1):18-36.

2. Thai QA, Raza SM, Pradilla G, Tamargo RJ. Aneurysmal rupture without subarachnoid hemorrhage: case series and literature review. Neurosurgery. 2005;57(2):225-9; discussion 225-9.

3. Abbed KM, Ogilvy CS. Intracerebral hematoma from aneurysm rupture. Neurosurg Focus. 2003;15(4):E4.

4. Sadato N, Numaguchi Y, Rigamonti D, Salcman M, Gellad FE, Kishikawa T. Bleeding patterns in ruptured posterior fossa aneurysms: a CT study. J Comput Assist Tomogr. 1991;15(4): 612-7.

5. Pasqualin A, Bazzan A, Cavazzani P, Scienza R, Licata C, Da Pian R. Intracranial hematomas following aneurysmal rupture: experience with 309 cases. Surg Neurol. 1986;25(1):6-17.

6. Tokuda Y, Inagawa T, Katoh Y, Kumano K, Ohbayashi N, Yoshioka $H$. Intracerebral hematoma in patients with ruptured cerebral aneurysms. Surg Neurol. 1995;43(3):272-7.

7. Lee GY, Lim SM, Kim Y. Hypertensive intracerebral hematoma after aneurysmal subarachnoid hemorrhage. J Clin Neurosci. 2007;14(12):1233-5.
8. Parizel PM, Makkat S, Jorens PG, Ozsarlak O, Cras P, Van Goethem JW, et al. Brainstem hemorrhage in descending transtentorial herniation (Duret hemorrhage). Intensive Care Med. 2002;28(1):85-8.

9. Pollock H, Hutchings M, Weller RO, Zhang ET. Perivascular spaces in the basal ganglia of the human brain: their relationship to lacunes. J Anat. 1997;191(Pt 3):337-46.

10. Zhang ET, Inman CB, Weller RO. Interrelationships of the pia mater and the perivascular (Virchow-Robin) spaces in the human cerebrum. J Anat. 1990;170:111-23.

11. Elster AD, Richardson DN. Focal high signal on MR scans of the midbrain caused by enlarged perivascular spaces: MRpathologic correlation. AJR Am J Roentgenol. 1991;156(1): 157-60.

12. Saeki N, Sato M, Kubota M, Uchino Y, Murai H, Nagai Y, et al. MR imaging of normal perivascular space expansion at midbrain. AJNR Am J Neuroradiol. 2005;26(3):566-71.

13. Kwee RM, Kwee TC. Virchow-Robin spaces at MR imaging. Radiographics. 2007;27(4):1071-86.

14. Yasargil MG, Kasdaglis K, Jain KK, Weber HP. Anatomical observations of the subarachnoid cisterns of the brain during surgery. J Neurosurg. 1976;44(3):298-302.

15. Wessels T, Moller-Hartmann W, Noth J, Klotzsch C. CT findings and clinical features as markers for patient outcome in primary pontine hemorrhage. AJNR Am J Neuroradiol. 2004;25(2): 257-60.

16. Wijdicks EF, St Louis E. Clinical profiles predictive of outcome in pontine hemorrhage. Neurology. 1997;49(5):1342-6.

17. Kim JS, Bae YH. Pure or predominant sensory stroke due to brain stem lesion. Stroke. 1997;28(9):1761-4.

18. Yasuda Y, Watanabe T, Tanaka H, Ogura A. Localizing value of bilateral cheiro-oral sensory impairment. Intern Med. 1998;37 (11):982-5 